\title{
Updates in Treatment Strategies for Hodgkin Lymphoma
}

\author{
Presented by Ranjana H. Advani, MD
}

\begin{abstract}
Several options are available for frontline treatment of advanced-stage Hodgkin lymphoma $(\mathrm{HL})$ and treatment of relapsed $\mathrm{HL}$, each with inherent advantages and disadvantages. Clinicians must balance risk with benefit for the individual patient. At the NCCN 2019 Annual Congress: Hematologic Malignancies, Dr. Ranjana H. Advani summarized the current frontline treatment options for advanced-stage HL and outlined novel and emerging agents that may be incorporated as therapy options for relapsed disease.
\end{abstract}

J Natl Compr Canc Netw 2019;17(11.5):1411-1413 doi: $10.6004 /$ jnccn.2019.5027

Given the myriad of treatment options available for frontline treatment of advanced-stage Hodgkin lymphoma (HL) and for relapsed HL, clinicians must evaluate the advantages and disadvantages of each regimen, balancing risk with benefit for the individual patient.

At the NCCN 2019 Annual Congress: Hematologic Malignancies, Ranjana H. Advani, MD, Saul Rosenberg Professor of Lymphoma, Stanford Cancer Institute, summarized current standard treatment options for classic HL and discussed the incorporation of novel therapeutic agents in the management of patients with advanced or relapsed/refractory disease. Dr. Advani also outlined potential strategies to consider when developing treatment plans for patients with HL, including prognostic markers and sequence of treatment. In addition, Dr. Advani noted that because most patients with HL survive, ${ }^{1}$ optimizing survivorship must be a priority in treatment selection.

Dr. Advani outlined the 3 suggested treatment options for advanced HL (stage III and IV) in the NCCN Guidelines. ${ }^{2}$ The first regimen, ABVD (doxorubicin/bleomycin/ vinblastine/dacarbazine), has been around for 30 years, is easy to use, and is effective in most patients. The second regimen, escalated BEACOPP (bleomycin/ etoposide/doxorubicin/cyclophosphamide/vincristine/ procarbazine/prednisone), increases progression-free survival (PFS) but at the cost of significant toxicity with no overall survival (OS) advantage. Finally, somewhere in between, said Dr. Advani, is the newcomer: brentuximab vedotin (BV) + AVD (doxorubicin/vinblastine/dacarbazine).

According to Dr. Advani, interim PET has emerged as a useful tool for distinguishing response to therapy. In this arena, 2 important questions are in focus: will modification of therapy based on interim PET results allow for selection of patients for treatment escalation or de-escalation, and will these modifications improve outcomes. With that in mind, said Dr. Advani, the past decade has been spent developing PET-adapted strategies that study how to select patients for therapy escalation or de-escalation.

"Basically, there are 2 strategies for initial therapy," she said. "You can start with ABVD and then escalate or de-escalate based on interim PET, or start with escalated BEACOPP and then do an interim PET and decide what to do after."

\section{Interim PET-Adapted Strategies}

As Dr. Advani reported, 3 large trials have evaluated the first approach: starting with ABVD for 2 cycles, performing an interim PET, and switching to escalated BEACOPP for patients with PET-positive disease. ${ }^{3-5}$ Depending on the trial and inclusion of patients with stage II bulky disease, PFS rates ranged from $75 \%$ to $87 \%$; however, OS was well above $90 \%$ in all 3 trials, and patients whose interim PET results were negative did well. Patients with interim PETpositive disease had PFS rates between $60 \%$ and $65 \%$ with excellent OS ( $\sim 90 \%)$.

"These data show that you can reserve the toxicity of escalated BEACOPP for a highly selected group of patients who have positive results on interim PET," said Dr. Advani, who noted that in another trial, patients with positive PET results underwent stem cell transplant and demonstrated very good outcomes, with a PFS of $75 \%{ }^{6}$

The other strategy for initial therapy involves starting patients on the more intensive BEACOPP regimen. $\mathrm{Pa}$ tients who have negative results on interim PET are reduced to ABVD, which is very well tolerated, whereas those with positive results remain on escalated BEACOPP.? As Dr. Advani reported, the results have been excellent, with standard and experimental arms almost completely overlapping. Patients with positive PET results after 4 cycles of escalated BEACOPP had the worst outcomes, irrespective of arm. 
"These interim PET-adapted strategies provide an opportunity to personalize treatment approach," said Dr. Advani, who noted that therapy de-escalation after negative PET results largely retains its efficacy, whereas therapy escalation after positive PET results shows improved disease control and might warrant a change in treatment. "However, there are prognostic factors other than PET that might affect outcomes, including stage IV or higher International Prognostic Scores."

\section{Brentuximab Vedotin}

Another treatment approach for advanced HL involves $\mathrm{BV}$, an anti-CD30 antibody-drug conjugate. BV is FDAapproved for relapsed HL after autologous hematopoietic stem cell transplant (HSCT), failure of 2 regimens in patients not eligible for transplant, and as consolidation for patients with high-risk HL after autologous HSCT. BV is also approved in the frontline setting in combination with AVD (BV-AVD) for stage III and IV HL. The latter indication was based on data from the phase III ECHELON-1 study, which randomized patients with newly diagnosed advanced HL to BV-AVD versus ABVD. ${ }^{8}$

As Dr. Advani reported, treatment with BV-AVD was associated with a $5 \%$ improvement in modified PFS but also higher rates of febrile neutropenia and neuropathy. The FDA indication approval recommends growth factor support with BV-AVD. ${ }^{8}$

The other issue to consider with these regimens is cost. Because of the growth factor requirement, said Dr. Advani, a 6-month treatment course with BV-AVD is $\$ 284,616$ versus only $\$ 3,648$ for ABVD. 9 "This gets back to the dilemma of therapy," said Dr. Advani. "Do you want better outcomes upfront or reduced toxicity? You really have to individualize therapy based on what your patient can tolerate and what resources you have available."

\section{Checkpoint Inhibitors and Biological \\ Parameters of High-Risk Disease}

Two checkpoint inhibitors, nivolumab and pembrolizumab, are also approved for relapsed disease, and investigators are exploring these agents in the frontline setting. The phase II CheckMate 205 trial showed that nivolumab monotherapy followed by nivolumab + AVD (Nivo-AVD) was well tolerated in newly diagnosed classic HL. ${ }^{10}$

"PFS with this combination has fallen to $83 \%$ at 2 years, and more follow-up is needed to see if it remains at that level," said Dr. Advani, who noted that a trial comparing BV-AVD versus Nivo-AVD in patients with newly diagnosed advanced-stage classic HL has already opened at a few centers across the country.

Researchers are also trying to identify subsets of patients with negative interim PET results who have poorer outcomes. Recent data have shown that $>25 \%$ expression of CD68, a diffuse versus scattered pattern of expression of PD-1 in the microenvironment, and STAT1-negativity in Hodgkin and Reed/Sternberg (HRS) cells are all associated with significantly worse PFS, even in patients who are interim PET-negative (64\% vs 95\%). ${ }^{11}$

"Maybe in time we'll be able to select patients upfront based on biologic parameters," Dr. Advani observed.

\section{Relapsed/Refractory Disease}

Despite the success seen with frontline therapies, however, between $10 \%$ and $30 \%$ of patients will develop primary refractory or relapsed disease, said Dr. Advani, who emphasized the importance of performing a biopsy on relapse. Standard therapy for patients with disease relapse is a second-line regimen followed by autologous HSCT, which leads to cure in approximately half of patients.

The combination of $\mathrm{BV}+$ nivolumab has also shown impressive results, said Dr. Advani, with the complete response rate at approximately $61 \% .^{12}$ Furthermore, patients who experienced complete response after nivolumab $+\mathrm{BV}$ and transplant had an estimated 2-year PFS of $96 \%$.

Finally, given the success of the BV/nivolumab combination, investigators are even exploring the addition of another checkpoint inhibitor, ipilimumab, which targets CTLA-4.

Regarding the combination of multiple targeted agents, "in a very small interim analysis, it appears that PFS may be in favor of the triplet versus the doublet, but time will tell," Dr. Advani concluded.

Disclosures: Dr. Advani has disclosed that she has received research

support from Agensys, Inc., Celgene Corporation, Forty Seven, Inc., Janssen Pharmaceutica Products, LP, Kura Oncology, Inc., Merck \& Co., Inc., Millennium Pharmaceuticals, Inc., Genentech, Inc./Roche Laboratories, Inc., Pharmacyclics, Regeneron Pharmaceuticals, Inc., Seattle Genetics, Inc. and serves as a scientific advisor for AstraZeneca Pharmaceuticals LP, Bayer HealthCare, Gilead Sciences, Inc., Kite Pharma, Kyowa Hakko Kirin Co. Ltd., Cell Medica, Genentech, Inc./Roche Laboratories, Inc., Seattle Genetics, Inc., and Takeda Pharmaceuticals North America, Inc.

Correspondence: Ranjana H. Advani, MD, Stanford Cancer Institute, 875 Blake Wilbur Drive, Stanford, CA 94305. Email: radvani@stanford.edu

\section{References}

1. SEER Cancer Stat Facts: Hodgkin Lymphoma. National Cancer Institute. Bethesda, MD. Available at: https://seer.cancer.gov/statfacts/html/ hodg.html. Accessed October 23, 2019.

2. Hoppe RT, Advani RH, Ai WZ, et al. NCCN Clinical Practice Guidelines in Oncology: Hodgkin Lymphoma. Version 2.2019. Accessed October 18, 2019. To view the most recent version, visit NCCN.org

3. Press OW, Li H, Schöder H, et al. US Intergroup trial of response-adapted therapy for stage III to IV Hodgkin lymphoma using early interim fluorodeoxyglucose-positron emission tomography imaging: Southwest Oncology Group S0816. J Clin Oncol 2016;34:2020-2027.

4. Johnson P, Trotman J, Federico M. Interim PET-CT scan in advanced Hodgkin's lymphoma. N Engl J Med 2016;375:999-1000. 
5. Gallamini A, Tarella C, Viviani S, et al. Early chemotherapy intensification with escalated BEACOPP in patients with advanced-stage Hodgkin lymphoma with a positive interim positron emission tomography/ computed tomography scan after two ABVD cycles: long-term results of the GITIL/FIL HD 0607 trial. J Clin Oncol 2018;36:454-462.

6. Zinzani PL, Broccoli A, Gioia DM, et al. Interim positron emission tomography response-adapted therapy in advanced-stage Hodgkin lymphoma: final results of the phase II part of the HD0801 study. J Clin Oncol 2016;34:1376-1385.

7. Casasnovas RO, Bouabdallah R, Brice P, et al. PET-adapted treatment for newly diagnosed advanced Hodgkin lymphoma (AHL2011): a randomised, multicentre, non-inferiority, phase 3 study. Lancet Oncol 2019;20:202-215.

8. Connors JM, Jurczak W, Straus DJ, et al. Brentuximab vedotin with chemotherapy for stage III or IV Hodgkin's lymphoma. N Engl J Med 2018;378:331-344.
9. Huntington SF, von Keudell G, Davidoff AJ, et al. Cost-effectiveness analysis of brentuximab vedotin with chemotherapy in newly diagnosed stage III and IV Hodgkin lymphoma. J Clin Oncol 2018 36:3307-3314.

10. Ramchandren R, Domingo-Domenech E, Rueda A, et al. Nivolumab for newly diagnosed advanced-stage classic Hodgkin lymphoma: safety and efficacy in the phase II CheckMate 205 study. J Clin Oncol 2019;37: 1997-2007

11. Agostinelli C, Gallamini A, Stracqualursi L, The combined role of biomarkers and interim PET scan in prediction of treatment outcome in classical Hodgkin's lymphoma: a retrospective, European, multicentre cohort study. Lancet Haematol 2016;3:e467-479.

12. Herrera AF, Moskowitz AJ, Bartlett NL, et al. Interim results of brentuximab vedotin in combination with nivolumab in patients with relapsed or refractory Hodgkin lymphoma. Blood 2018;131:1183-1194. 